February 18, 2003

CBN 03-1

\title{
CHARACTERISTICS OF GRADIENT UNDULATOR ${ }^{1}$
}

\author{
A. Mikhailichenko
}

Cornell University, LEPP, Ithaca NY 14853

Undulator/wiggler having the same polarities of magnetic field in opposing though medial plane poles can be used for generation radiation which intensity is a function of the beam size and displacement. Characteristics of such device analyzed here analytically and by tracking.

\section{INTRODUCTION}

Utilization of a quadrupole wiggler is an interesting option for broadening the bandwidth of pick-ups in fast feedback systems [1] and for beam alignment [2]. Such a wiggler, which is a sequence of ordinary focusing and defocusing quadrupoles, generates a radiation, which depends on the beam shape and position.

Although in such system radiation for two transverse coordinates can be easily distinguished by polarization, desire to have sensitivity to one coordinate only is always an option. In [3] a device, called gradient undulator, was described for the first time. Practical utilization of this device suggested in [4].

Here we will concentrate our attention on practical aspects of design and properties of magnetic fields in gradient undulator. The test was also included trajectories analyses, which was carried out numerically on the basis of real 3D model. Knowing the real trajectory allows finding radiated field instantly in principle.

\section{ANALYTICAL CONSIDERAIONS}

Let us consider first some simple aspects of symmetry of gradient undulator/wiggler in comparison with usual dipole wiggler; both are represented in Fig.1.
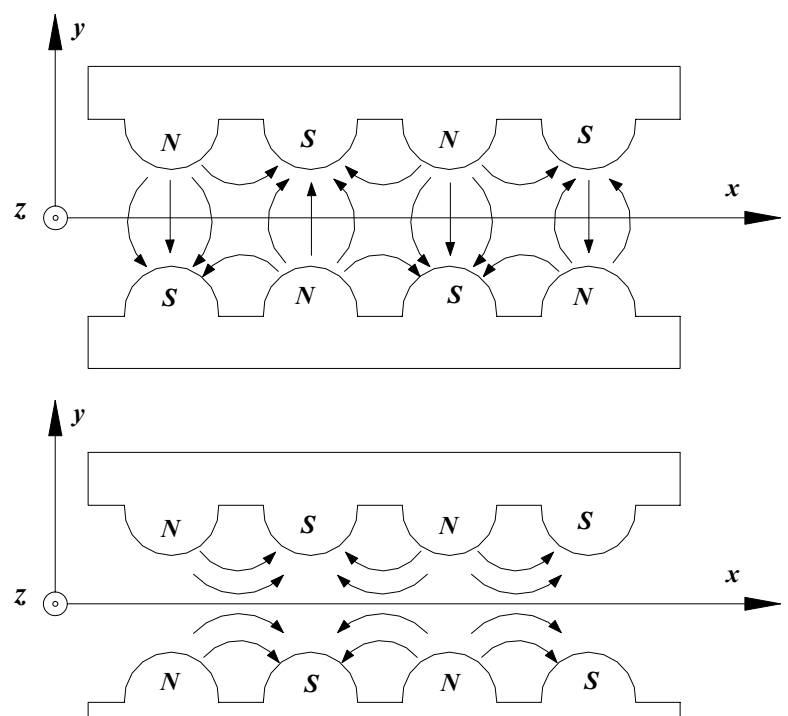

FIGURE 1: Dipole wiggler, upper one and gradient undulator/wiggler, lower. Beam is moving along $x$-axis. In normal to the drawing's plane direction, $z$-direction, the poles are much longer, than period in $x$ direction.

\footnotetext{
${ }^{1}$ Electronic version is available at http://www.lns.cornell.edu/public/CBN/2003/CBN03-1/CBN03 1.pdf
} 
One can see, first that in dipole wiggler the fields between poles represent a quadrupole type as functions of $x$ and $y$ with longitudinal axes of these quadrupoles oriented in $z$ direction. This means, that $x$ component of the field is absent at the center plane $\{x, z\} . B_{x}(x, y)$-component is a linearly growing function of $y . B_{y}(x, y)$ is zero at the places between the poles and reaches its maximal value in plane $\{x, z\}$ below the pole. So the particle moves across this set of quadrupoles experiencing focusing from these quadrupoles proportional to the wiggling angle, $\alpha(x, y) \cong e B_{y}(x, y) \cdot \lambda_{w} / m c^{2} \gamma \equiv K / \gamma$, and $2 \pi \lambda_{w}$ stands for the wiggler period. Motion is a pretty much sinusoidal one. Longitudinal field are increasing linearly in $y$ direction, while wiggling amplitude remains the same. So, the mechanism of focusing in $y$ direction-is in bending by longitudinal fields, what is a quadrupole type here. Gradient can be estimated as $G \cong B_{y} /\left(\lambda_{w} / 4\right)=\frac{2}{\pi} B_{y} / \lambda_{w}$, but effective gradient acting at the particle's trajectory is $G_{\text {eff }} \cong \alpha \cdot G=\frac{2}{\pi} \frac{e B_{y}^{2}}{m c^{2} \gamma} \equiv \frac{2}{\pi} \frac{B_{y}^{2}}{(H R)}$, where $(H R)$ stands for magnetic rigidity. As the passing over the pole region, particle changes its angle too, effective action over whole dipole wiggler is $\int G_{e f f} d x \cong \frac{1}{2} G_{e f f} \cdot L$, where $L$ is total wiggler length.

In gradient wiggler, the longitudinal field $B_{x}(y)$ has nonzero value at the plane $\{x, z\}$, but now wiggling amplitude linearly increasing with $y$ coordinate following $B_{y}(y)$.

Ideal dipole wiggler must have appropriate profile of quadrupoles to reduce appearance of undesirable harmonics. For wigglers with superconducting windings, the iron is saturated typically, so this kind of shaping is not much practical. From Fig. 1 it is clear that for dipole wiggler with infinitely wide poles, function $B_{y}(x)$ and its derivatives describe all 3D fields around. In gradient wiggler this can be done with knowing $B_{x}(x)$.

Let us make first, some analytical representations for the fields of gradient wiggler, using its specific symmetry. The mostly general form for the fields of gradient wiggler, having wide poles (width in $z$ direction is much bigger, than period) has the form

$$
B_{x}(x, y)=\sum_{k=0}^{\infty} B_{k}(x) \cdot y^{2 k}, B_{y}(x, y)=\sum_{k=0}^{\infty} A_{k}(x) \cdot y^{2 k+1},
$$

where $A_{k}(x), B_{k}(x)$-some smooth functions, which need to be determined. Using Maxwell's equation $\operatorname{div} \vec{B}=\frac{\partial B_{x}}{\partial x}+\frac{\partial B_{y}}{\partial y}=0$ one can obtain from (1) $\sum_{k} y^{2 k} \cdot\left\{B_{k}^{\prime}(x)+(2 k+1) \cdot A_{k}(x)\right\} \equiv 0$, or $A_{k}(x)=-\frac{1}{2 k+1} \cdot B_{k}^{\prime}(x)$. Second equation, $\left.\operatorname{rot} \vec{B}\right|_{z}=\frac{\partial B_{x}}{\partial y}-\frac{\partial B_{y}}{\partial x}=0$ yields the following condition

$$
\sum_{k}\left\{2 k \cdot y^{2 k-1} \cdot B_{k}(x)+\frac{1}{2 k+1} B_{k}^{\prime \prime}(x) \cdot y^{2 k+1}\right\}=0 .
$$

Equalizing terms with the same power, one can obtain recurrent formula

$$
2 k \cdot B_{k}(x)+\frac{B_{k-2}^{\prime \prime}(x)}{2 k-1}=0 .
$$


In it's turn, (3) yields the following

$$
B_{k}(x)=\frac{(-1)^{k}}{(2 k) !} B^{(2 k)}(x),
$$

where function $B(x) \equiv B_{0}(x)$ defines profile of longitudinal field at the axis $x$ and $B^{(2 k)} \equiv \frac{\partial^{2 k} B(x)}{\partial x^{2 k}}$ Substitute (4) into (1) we are coming to the analytical field expression for gradient wiggler as the following

$$
\begin{gathered}
B_{x}(x, y)=\sum_{k=0}^{\infty} \frac{(-1)^{k} y^{2 k} B^{(2 k)}(x)}{(2 k) !}, \\
B_{y}(x, y)=-\sum_{k=0}^{\infty} \frac{(-1)^{k} y^{2 k+1} B^{(2 k+1)}(x)}{(2 k+1) !}
\end{gathered}
$$

For demonstration, fields (5) can be represented in lowest order as the following

$$
\begin{gathered}
B_{x}(x, y)=B(x)-\frac{y^{2}}{2} B^{\prime \prime}(x)+\frac{y^{4}}{2 \cdot 12} B^{(I V)}(x)-\ldots \\
B_{y}(x, y)=-y \cdot B^{\prime}(x)+\frac{y^{3}}{2 \cdot 3} B^{\prime \prime \prime}(x)-\frac{y^{5}}{5 !} B^{(V)}(x)-\ldots
\end{gathered}
$$

One can see from here, that gradient in $y$ direction associated with $B^{\prime}(x)$. For example if dependence in $x$ direction is periodic, $B(x)=B_{0} \operatorname{Cos} \frac{x}{\lambda_{w}}$, the last determines the gradient in $y$ direction as

$$
G(x) \equiv \frac{\partial B_{y}}{\partial y}=-B^{\prime}(x)=\frac{B_{0}}{\lambda_{w}} \operatorname{Sin} \frac{x}{\lambda_{w}}
$$

So we are coming to fundamental conclusion for this type of wiggler, that the gradient is proportional to the longitudinal field and reciprocally proportional to the period. This relation does not depend on hardware details, such as saturation of yoke iron and so on. We will see lower, than numerical calculations in realistic system confirm (7). As it could be seen from (6) condition $\operatorname{div} \vec{B}=0$ and $\operatorname{rot} \vec{B}=0$ satisfied in every order of $y$.

It is interesting that for dipole wiggler with infinitely wide poles formulas for the fields can be represented in form (5), but now $B(x)$ defines the transverse dipole field and $B_{x}$ needs to be replaced by $B_{y}$ and vice versa $[5,6]$.

If the field is time dependent, then the following substitution needs to be done in all formulas [6]

$$
\frac{\partial^{2 k} B(x)}{\partial x^{2 k}} \rightarrow\left(\frac{\partial^{2}}{\partial x^{2}}-\frac{1}{c^{2}} \frac{\partial^{2}}{\partial t^{2}}\right)^{k} B(x, t)
$$

where $c$ stands for the speed of light. Odd derivative obtained by taking integral over $x$ in this combination. 
Of cause, for realistic situation, when poles are not wide, $z$ dependence of fields manifests itself. Mostly general form for potential of magnetic field in this case can be represented as the following [6]

$$
W=\left\{\int B(x) d x-\frac{\mid \xi^{2} B^{\prime}(x)}{4}+\frac{\mid \xi^{4} B^{m}(x)}{32 \cdot 2}-\cdots\right\}-i \sum_{m=1}^{\infty} \frac{\xi^{m} e^{-i m q_{0}}}{m}\left\{G_{m-1}(x)-\frac{\mid \xi^{2} G_{m-1}^{m}(x)}{4(m+1)}+\frac{\mid \xi^{4} G_{m-1}^{I V}(x)}{32(m+1)(m+2)}-\cdots\right\}
$$

where $\xi=z+i y$, and derivatives are taken along longitudinal axes $x$, and $m$ numerates multipoles and $\varphi_{0}=\pi / 4$ reflects the pole orientation (skew quad type). For simplicity in definition of coefficients, the terms responsible for longitudinal component separated in first brackets. The multipoles defined by theirs angular dependence. In case of dipole or gradient wigglers with sinusoidal dependence along longitudinal axes, angular dependence is not associated with the transverse one due to the presence of derivatives. Not all terms are present here for pure quadrupole symmetry remaining the only ones with $m=2,6,10$ and so on $(\equiv 2 \cdot(2 k+1), k=0,1,2, \ldots)$. Magnetic field can be expressed trough this potential as

$$
\bar{B}=B_{z}-i B_{y}=\frac{\partial W}{\partial \xi}+\frac{\partial W}{\partial \bar{\xi}}, \quad B_{x}=\operatorname{Re} \int\left(\frac{\partial^{2} W}{\partial x^{2}}\right) d x
$$

We will not develop this subject here, limiting our interest by (5) and coming to next topic.

\section{HARDWARE ASPECTS}

Let us consider now more or less realistic model of such a wiggler. Orientation of the poles is represented in Figs.1,2. This model has not associated with any specific project, but taken for example only.

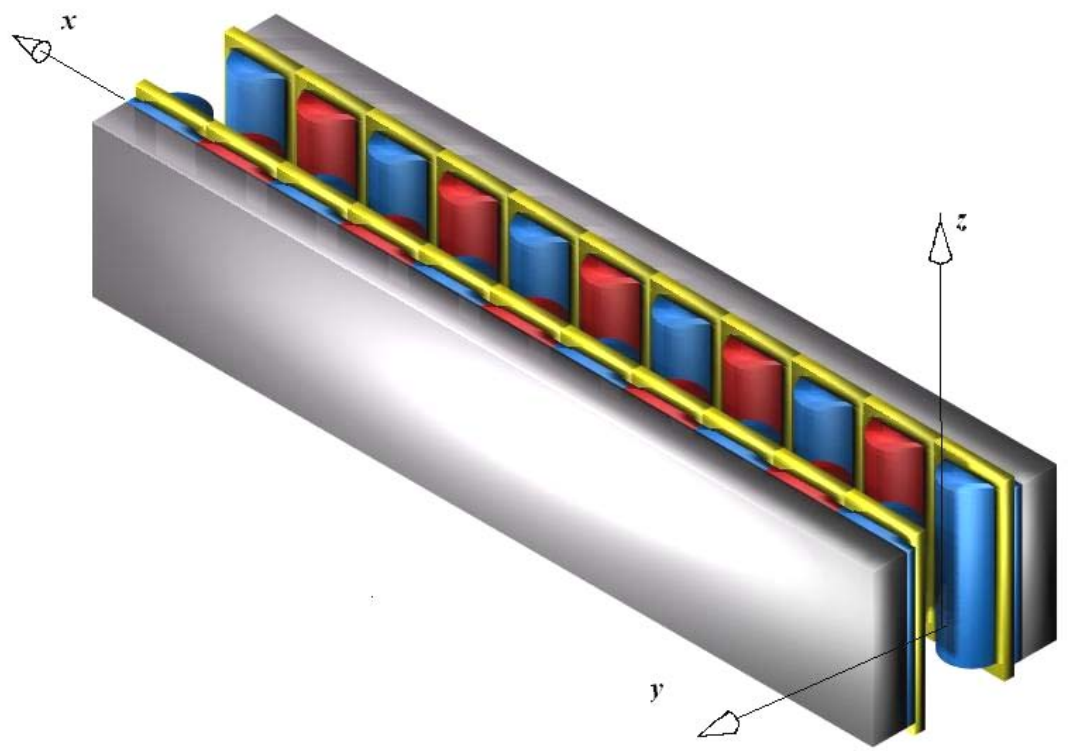

FIGURE 2(Color): View to the gradient wiggler model. Particles supposed to move along $x$. Pole size in z direction

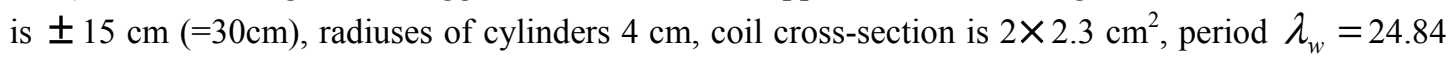
$\mathrm{cm}$, gap between poles $\pm 3.5 \mathrm{~cm}$. 
This wiggler is not sensitive to $z$ - position of the particle, giving linear dependence in radiated field across $y$ direction. Radiation in this wiggler is polarized along $z$-axis.

Yoke modeled by soft Steel 1010. Total current running in central coil is $60 \mathrm{kA}-\mathrm{Turns}$, what suggests utilization of SC windings. All field calculation done with 3D code MERMAID, Vectorial post-processing done with MATHEMATICA.

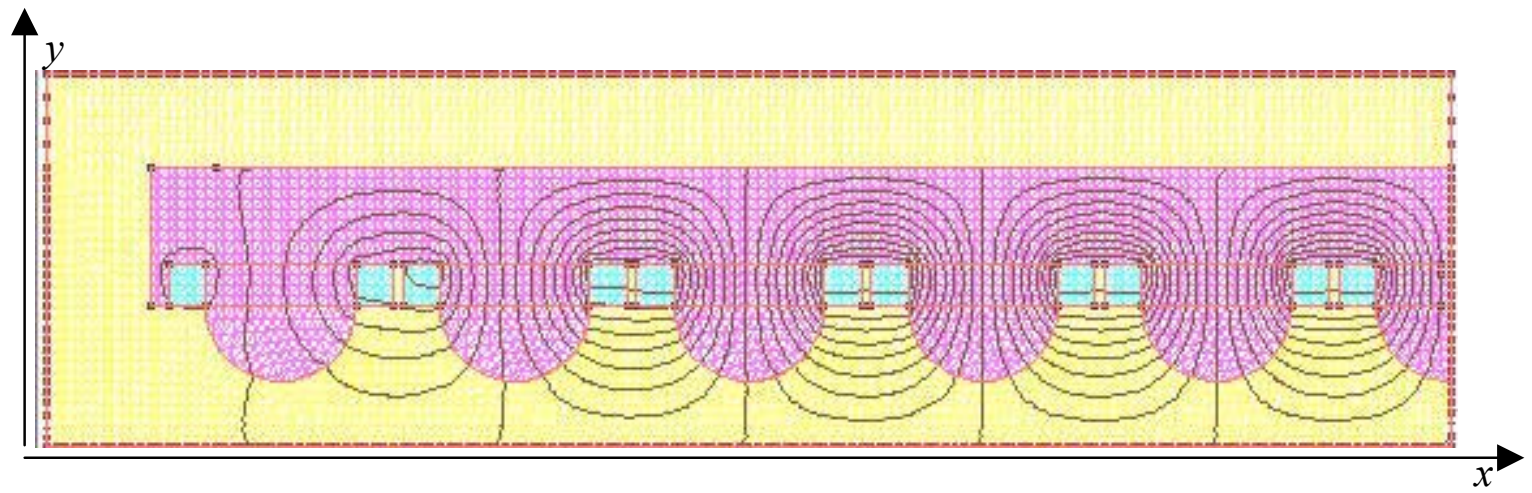

FIGURE 3(Color): Lines of magnetic field in central plane of wiggler, $z=0$. Half of the wiggler is shown; right side of the plot is a plane of symmetry along $x$. Radiuses of the poles are $4 \mathrm{~cm}$, period $-25 \mathrm{~cm}$. Gap between coils $0.5 \mathrm{~cm}$. Length of the pole in $z$ direction is $\pm 15 \mathrm{~cm}$. Particles are moving from the left side. $\{x z, y=0\}$ represents medial plane.

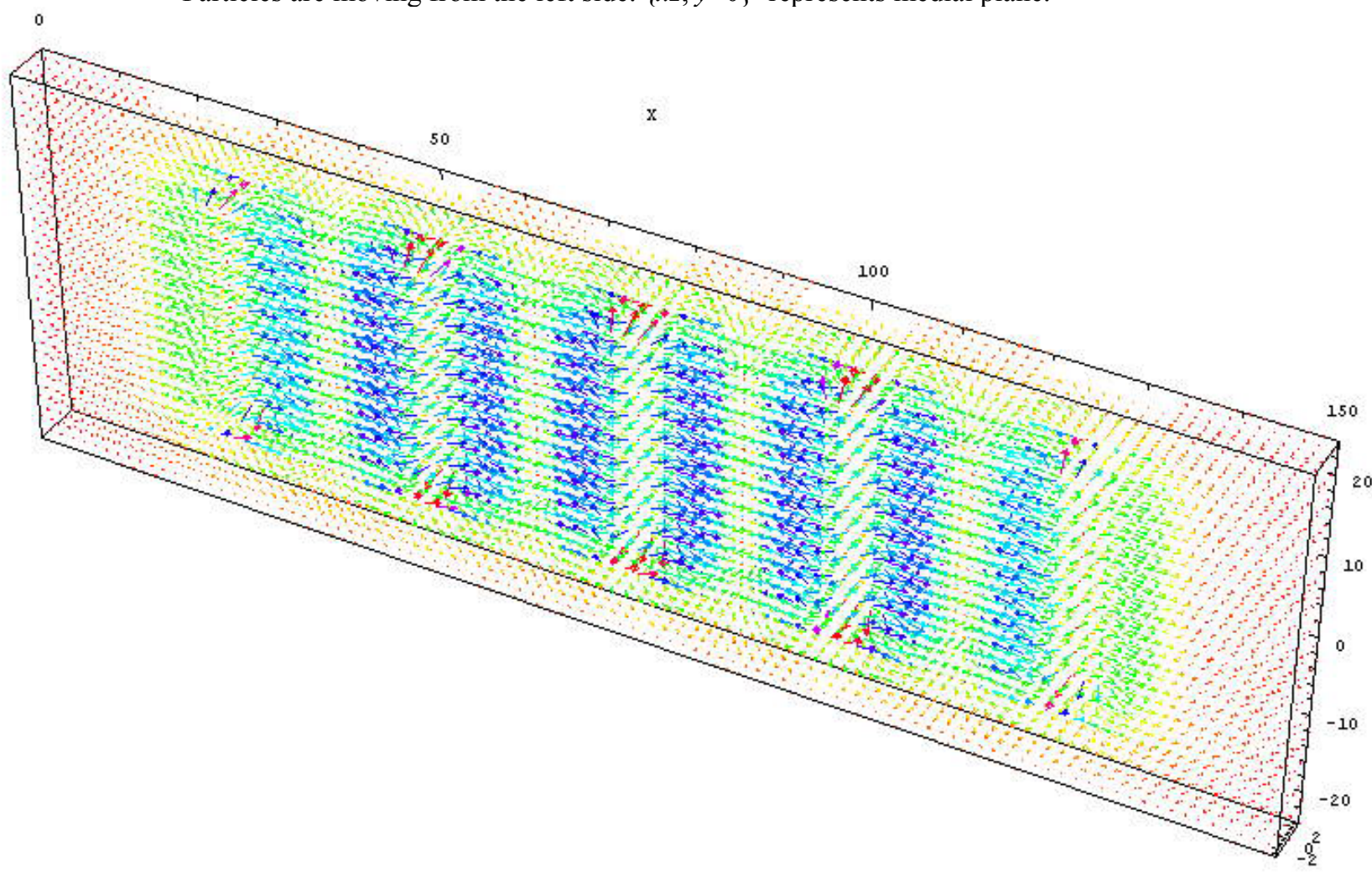

FIGURE 4(Color): Vectorial representation of the fields in the gap of 11-pole gradient wiggler inside the $3 \mathrm{D}$ volume $\{\mathrm{x}=0-150 ; \mathrm{y}= \pm 3 ; \mathrm{z}= \pm 24\} \mathrm{cm}$. Medial plane of the wiggler $\{\mathrm{x}, \mathrm{z}\}$ runs at $\mathrm{y}=0$. 
What is interesting about (7) is that at first look, the gradient defined by the distance between poles and period is not playing any role at all. One can see however from Fig.3, that field $B_{y}$ below the pole defined by the flux, what is coming from sides. The last one, of cause, depends on the wiggler period and $B_{y}$.

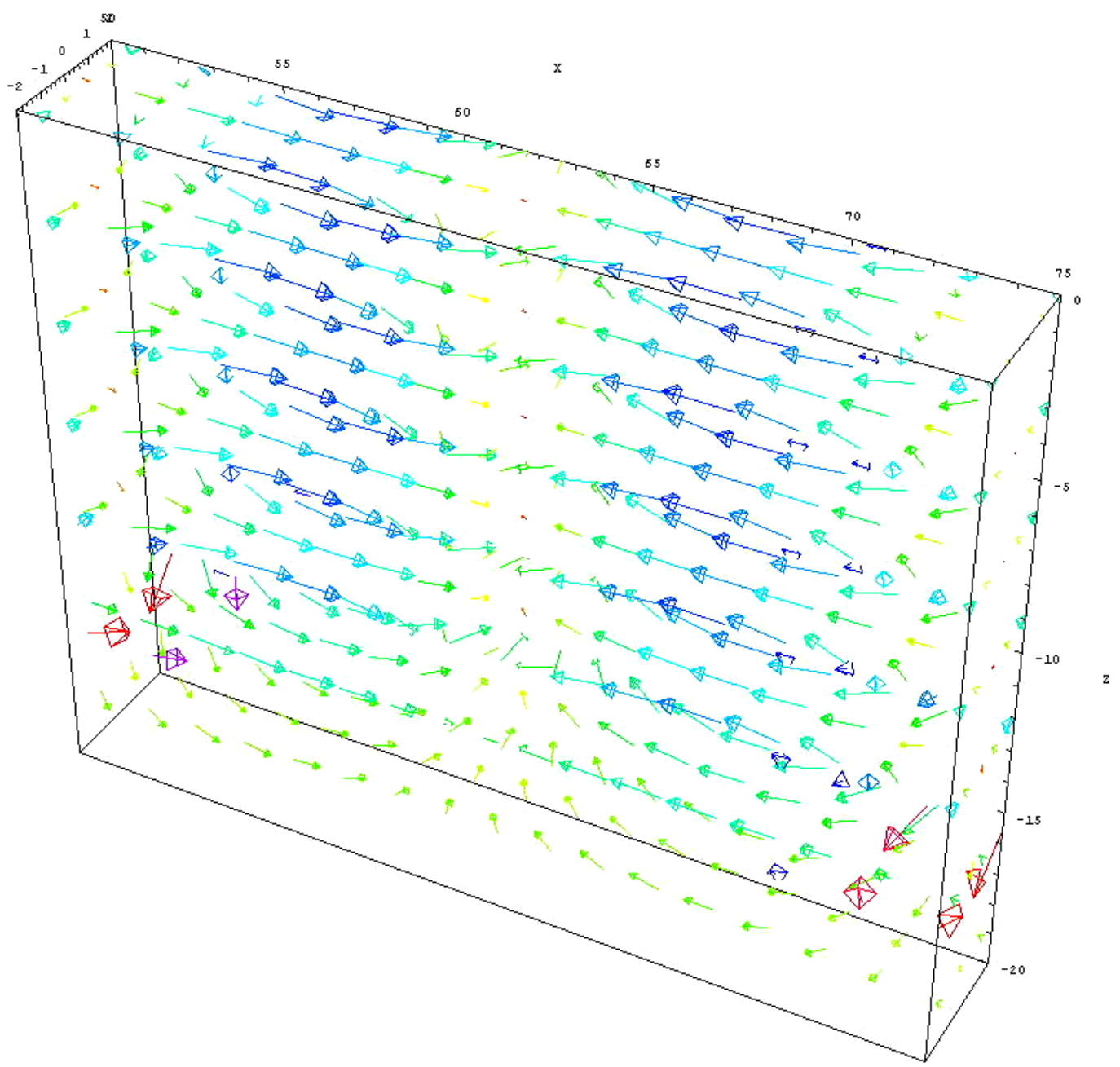

FIGURE 5(Color): Fragment of previous picture with coordinates $\{x=50-75 ; y= \pm 5, z=0-25\} \mathrm{cm}$, i.e. close to the central region of magnet.

First arises the question about the pole shape. The field must be as strong as possible for higher sensitivity. In case of flat poles this will produce an increase of field values at the side tips of the poles braking pure sinusoidal longitudinal field dependence. One can see, that as two opposing poles generating linear field dependence, they must have a shape close to hyperbolic. We used poles with cylindrical shape, however.

Wiggler is focusing towards medial plane $\{\mathrm{x}, \mathrm{z}\}$ in $\mathrm{y}$-direction. This focusing is typical for any wiggler and is proportional to the associated angle of wiggling $\alpha \cong K / \gamma$, with $K$-factor depending on vertical position itself. Effective gradient experienced by particle passing one pole gap is going to be $\int G(x, y) d x \equiv \frac{1}{2} \alpha B(y)$. In its turn, $B(y) \cong k \cdot y$. 
Calculations were carried for yoke with soft steel 1010 so the numbers are realistic and giving impression about whole picture. Current running in central pole is $60 \mathrm{kATurns}$ total. Two end poles from each side adjusted for zero offset of trajectory, see lower.

Coil cross section is a rectangle with dimensions $\{x \times y\}=\{2 \times 2.5\} \mathrm{cm}^{2}$. Yoke plate ends at $y=15$ $\mathrm{cm}$ from medial plane $y=0$.

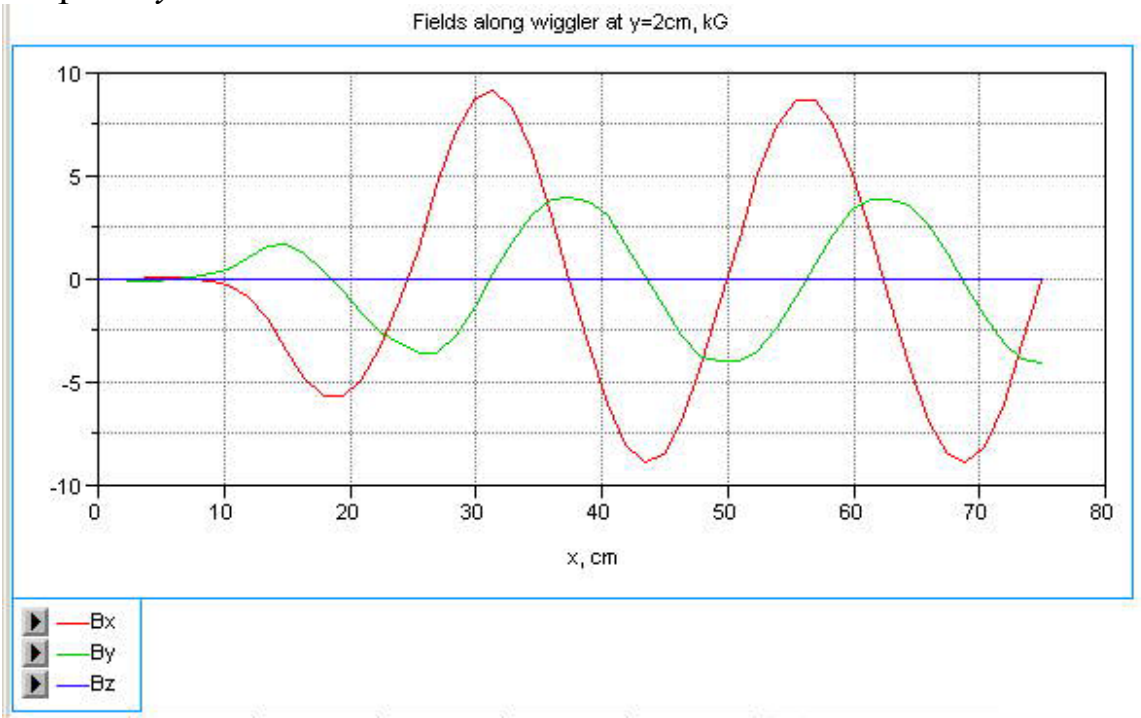

FIGURE 6(Color): Field graphs for $y=+2 \mathrm{~cm}, \mathrm{z}=0$. Half wiggler is shown as in Figure 3. Center is at the right side of the plot at $75 \mathrm{~cm}$. $B_{y}$ comes to it's maximum, $B_{x}$, indeed comes to zero. Basically $B_{\mathrm{z}}=0$ along this axis as it must be according to symmetry properties.

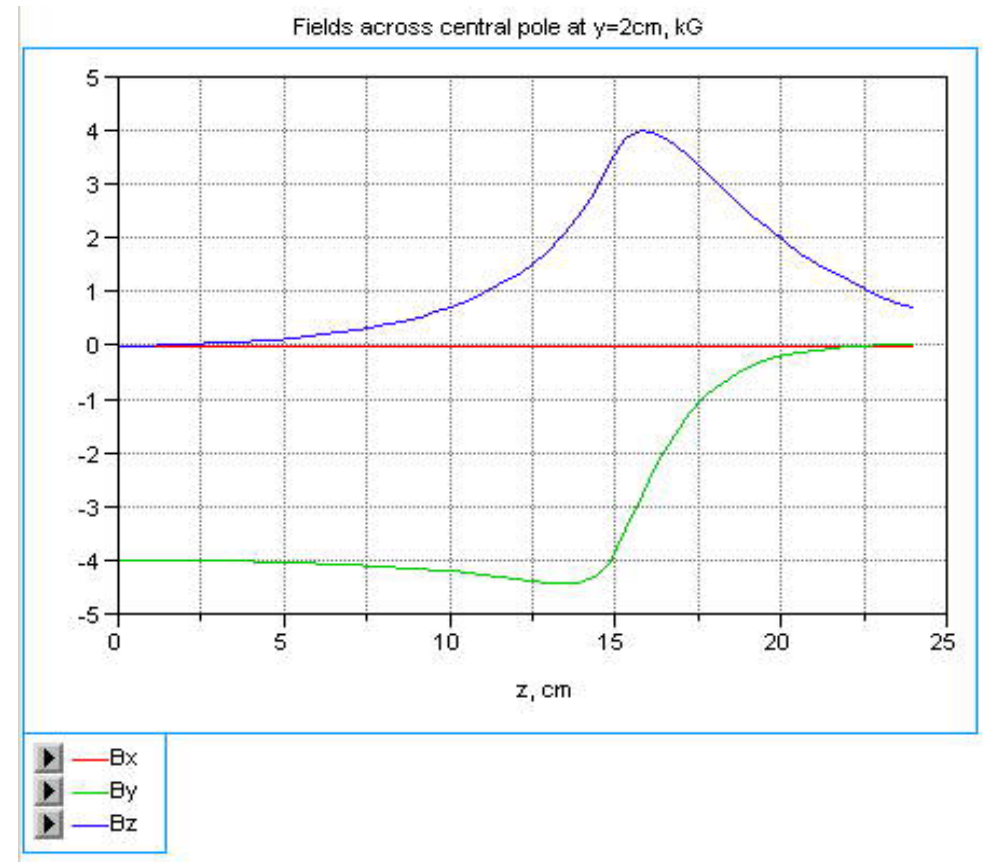

FIGURE 7 (Color): Fields across central pole, starting from $\mathrm{z}=0, \mathrm{y}=2 \mathrm{~cm}$. Material of yoke St1010. Points at $\{\mathrm{x}=75$, $\mathrm{y}=2, \mathrm{z}=0-24\} \mathrm{cm}$. The increase in $B_{\mathrm{y}}$ component (lower curve) at the side of the pole explained by the flux concentration here. The flux expelled from central region escapes from the sides. This effect also generates $B_{z}$ component. 
The component $B_{y}$ as a function position towards the pole tip is represented in Fig. 8 .

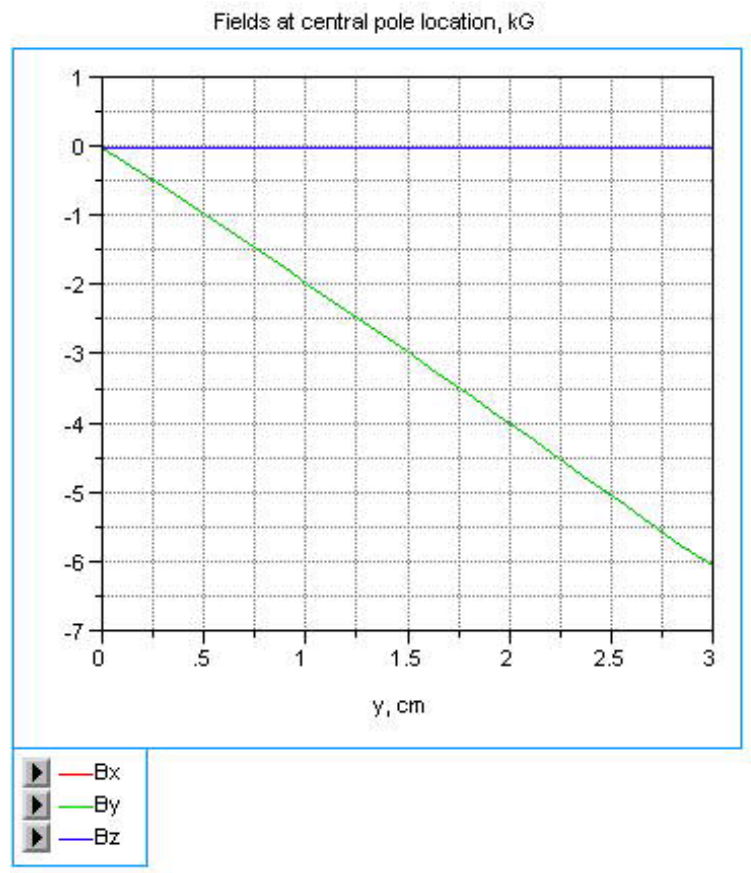

FIGURE 8(Color): Fields at the line, starting from $\mathrm{y}=0 ; \mathrm{z}=0, \mathrm{x}=75 \mathrm{~cm}$ (middle of central pole) towards the central pole. Pole iron begins at $3.5 \mathrm{~cm}$. Basically the only $B_{y}$ is present.

Bx between poles, starting from medial plane, $\mathrm{kG}$

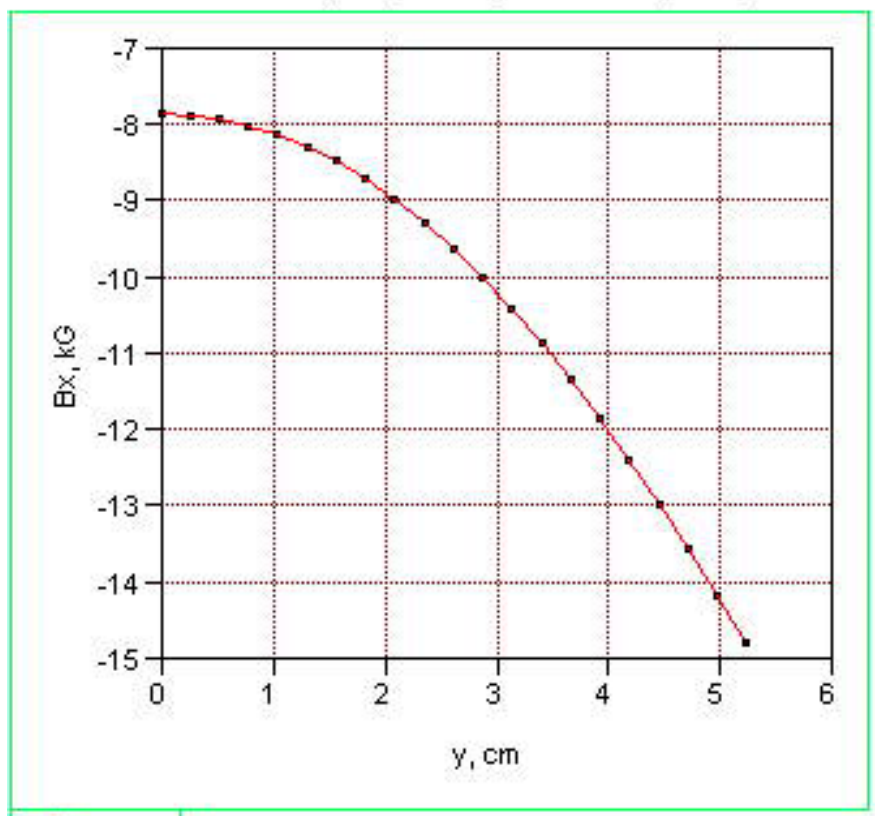

$\mathrm{Bx}, \mathrm{kG}$

FIGURE 9: Fields between poles: the central one and next to it as functions of $y$. Basically only $B_{\mathrm{x}}$ component is present, as it must be according to symmetry properties. Next to the central pole gap was chosen for representation. 
Field $B_{x}$ from Fig.9 as a function of $y$ coordinate can be represented as the following

$$
B_{x}(y)=-7.82+0.0786 \cdot y+0.27 \cdot y^{2}+0.0031 \cdot y^{3}+\ldots
$$

One can see, that this is a quadratic dependence mostly as it must be from symmetry considerations.

From Fig. 8 one can calculate gradient $G_{1} \cong-2 k G / \mathrm{cm}$. From Fig.9, longitudinal field $B_{x} \cong 7.82 \mathrm{kG}$. Taking into account, that period is $24.8 \mathrm{~cm}$, or $\lambda_{w} \cong 24.8 / 2 \pi \cong 3.95 \mathrm{~cm}$, one can obtain $B_{x} / \lambda_{w}=7.82 / 3.95=1.98$ in good agreement with prediction (7).

\section{TRAJECTORIES}

Formally, as formulas for magnetic field is known, (5), it is possible to make analytical calculations of particle's trajectory. We will continue here our numerical exercises, however. Trajectories calculated numerically by using code UMKA [7] on the basis of MERMAID 3D field calculations.

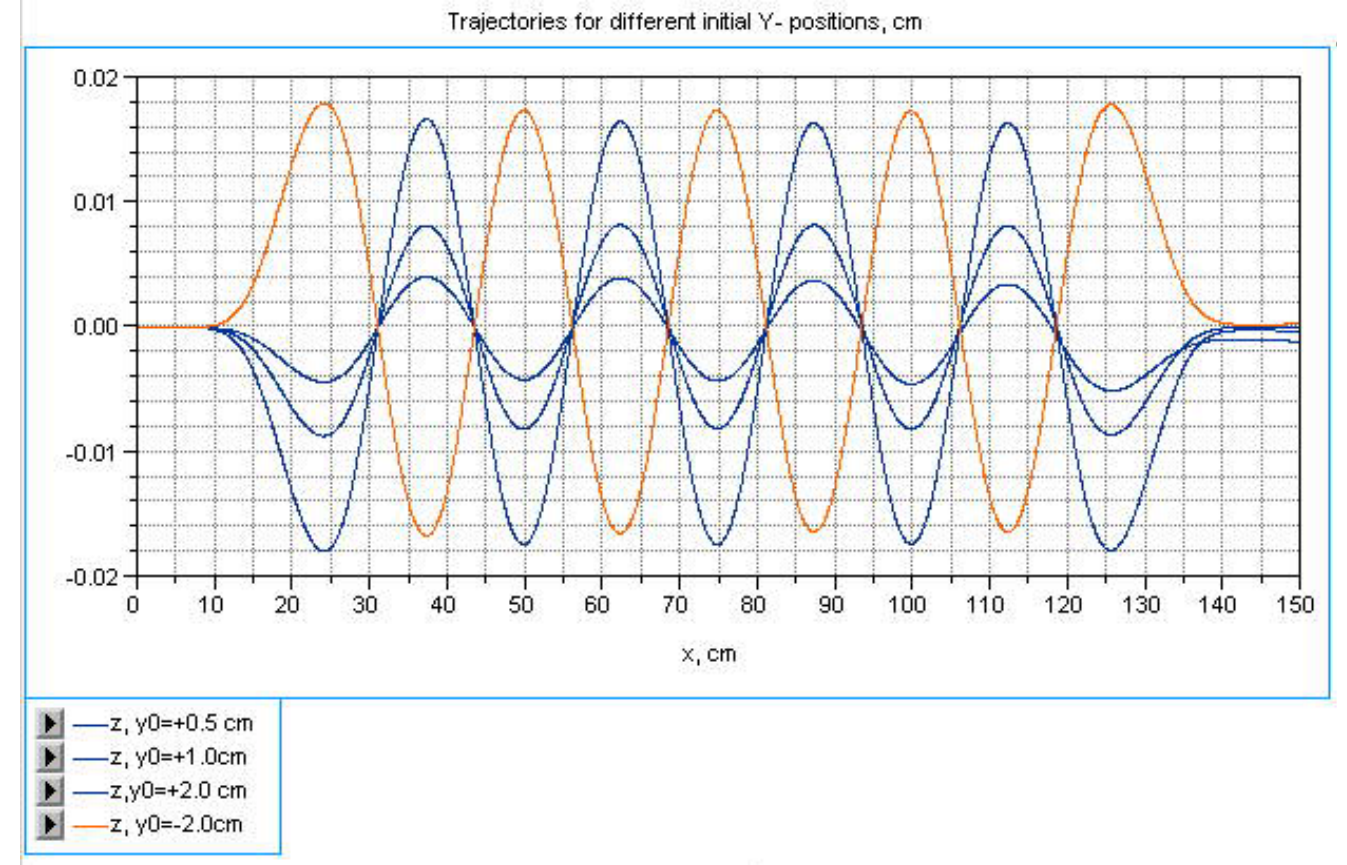

FIGURE 10(Color): Trajectories for different initial positions in $\mathrm{Y}$ direction. There are shown ones for $y_{0}=+0.5 \mathrm{~cm},+1.0 \mathrm{~cm},+2.0 \mathrm{~cm},-2.0 \mathrm{~cm}$. The last one corresponds the start point below median plane. Beam energy $2 \mathrm{GeV}$.

One can expect, as the field in vertical direction is linearly varying, trajectories with zero angle and displacement at output for one vertical input will have different resulting kicks and angles for other vertical input. This is seen in Fig.10, where particle with input $y_{0}=0.5 \mathrm{~cm}$ has displacement $\sim 10 \mu \mathrm{m}$ at the exit. 


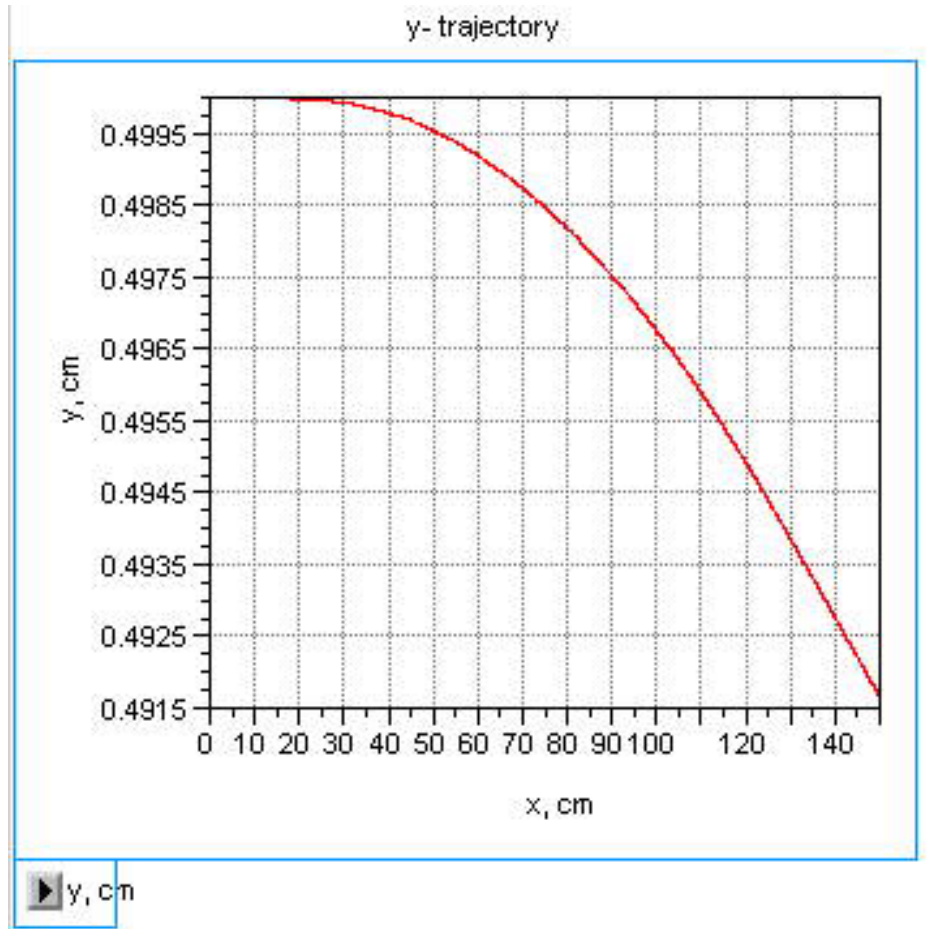

FIGURE 11: Trajectory for start position at $y=0.5 \mathrm{~cm}$. Beam energy $2 \mathrm{GeV}$.

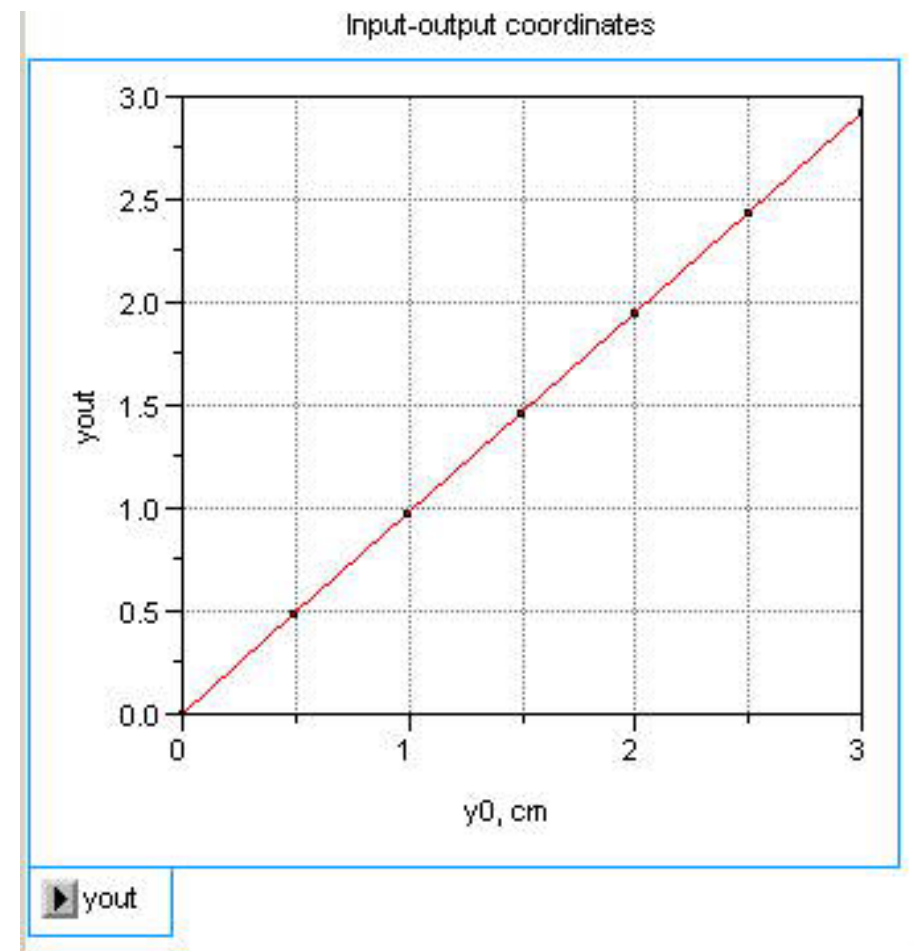

FIGURE 12: $y$-coordinate at the out as a function of input one. 


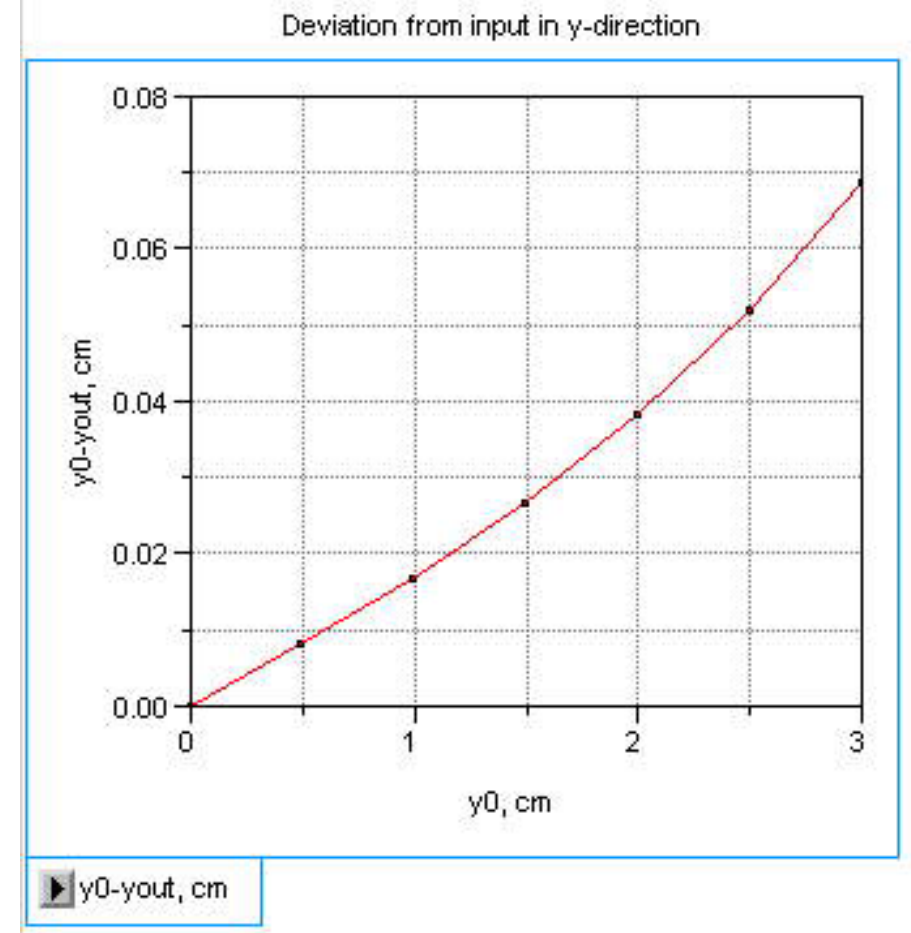

FIGURE 13: The difference between initial and final coordinates. Manifestation of nonlinear focusing is visible here.

Dependence of deviation in $y$-direction from initial coordinate, represented in Fig. 10, right can be expressed analytically as the following

$$
\Delta y\left(y_{0}\right) \equiv y_{0}-y_{\text {out }}\left(y_{0}\right)=0.0162 \cdot y_{0}+7.54 \cdot 10^{-4} \cdot y_{0}^{3} \text {. }
$$

So for input coordinate at the edge of aperture, $y_{0}=3 \mathrm{~cm}$, the fraction of nonlinear kick to the linear one goes to be $\delta=\frac{7.54 \cdot 10^{-4} \cdot 3 \cdot 9}{0.0162 \cdot 3} \equiv \frac{7.54 \cdot 9}{162}=41 \%$. However this ratio becomes drastically lower for small deviations from medial plane.

So one can see, that this type of wiggler can be used for fine-tuning of dynamic aperture of damping ring for linear collider, typically overloaded by dipole wigglers.

The set with only a pair of poles can be used for transformation of kick orientation. Really, particles directed towards one or another pole receive the kick in orthogonal direction depending to which pole they were deflected primary.

\section{RADIATION AND ACTION TO THE PARTICLE}

As trajectory $z(x)$ is known, this allows calculation of second derivative $\dot{\vec{v}} \cong \ddot{z}(x=c t)$ and hence, electric field as [8] $\vec{E}(t)=\frac{e}{c^{2}} \frac{\mid \vec{R} \times\lfloor(\vec{R}-\vec{\beta} R) \ddot{z}\rfloor}{(R-\vec{R} \cdot \vec{\beta})^{3}}$, where $\vec{\beta}=\vec{v} / c$ and $R$ is the distance to observer and derivatives are taken over time structure at the moment of radiation. So basically to obtain 
time structure at the observation point one needs to take second derivative with graph in Fig.10 and shrink this curve by Doppler factor $1 /(1-\vec{n} \vec{v})$, where $\vec{n}$ is unit vector to the observer. One can see that a single particle spectrum is the same as in a dipole wiggler with the same trajectory and formulas are the same (see for example [4]).

Let us see however, what are limitations for the width of spectrum might be. Evidently, the frequency of radiation

$$
\omega=\frac{c / \lambda_{w}}{1-\vec{n} \vec{v}} \cong \frac{2 \gamma^{2} \cdot c / \lambda_{w}}{1+\gamma^{2} \vartheta^{2}+K^{2} / 2}
$$

is a function of magnetic field through $K=\frac{e B_{y}(y) \lambda_{w}}{m c^{2}}=93.4 \cdot B_{y}(y)[$ Tesla $] \cdot 2 \pi \lambda_{w}[m], \vartheta$ is an angle to observer (from $x$-direction). So with variation of $y, K$ factor also changes, as $B_{y}(y)=B_{0} \cdot y / \lambda_{w}$, see formula (6). If one restricts the width at the level $\sim 50 \%$ from maximal, then $K^{2} / 2 \leq 1$, and hence, $K=\frac{e B_{0} y}{m c^{2}} \leq \sqrt{2}$. This limits either $B_{0}$ for given amplitude $y$ or amplitude, if $B_{0}$ is given. For the wiggler with given field at the axis $B_{0}$ the broadening will be less than $50 \%$ for amplitudes

$$
y \leq \sqrt{2} \cdot \frac{m c^{2}}{e B_{0}} \equiv \sqrt{2} \cdot \lambda_{w} \frac{m c^{2}}{e B_{0} \lambda_{w}}=\frac{\sqrt{2} \cdot \lambda_{w}}{K_{0}},
$$

where $K_{0}=e B_{0} \lambda_{w} / m c^{2}$. For the wiggler considered above, $B_{0} \sim 0.8 T, 2 \pi \lambda_{w} \cong 0.25 \mathrm{~m}$, $K_{0} \cong 18.7$ and amplitude must be $y \leq 0.3 \mathrm{~cm}$ i.e. below $3 \mathrm{~mm}$. For narrower width, the amplitude must be restricted proportionally.

Energy of the photon defined

$$
\hbar \omega=\frac{c \hbar / \lambda_{w}}{1-\vec{n} \vec{v}} \cong \frac{2 \cdot c \hbar / \lambda_{w} \gamma^{2}}{1+\gamma^{2} \vartheta^{2}+K^{2} / 2}=\hbar \omega_{\max } \frac{1}{1+\gamma^{2} \vartheta^{2}+K^{2} / 2},
$$

where maximal energy defined as

$$
\hbar \omega_{\max }=2 \hbar \gamma^{2} \frac{c}{\lambda_{w}} \equiv 2 \hbar \gamma^{2} \Omega_{w} \equiv 4 \pi \gamma^{2} \frac{\hbar c}{\lambda_{w}} .
$$

Taking into account that conversion constant $\hbar c \cong 197.33 \mathrm{MeV} \cdot \mathrm{fm}$, and for the wiggler discribed, $\lambda_{w}=0.2484 m \cong 0.248 \cdot 10^{15} \mathrm{fm}$, for $\gamma=4000(\sim 2 \mathrm{GeV}), \gamma^{2}=1.6 \cdot 10^{7}$,

$$
\hbar \omega_{\max } \cong 4 \pi 1.6 \cdot 10^{7} \frac{197.33}{0.248 \cdot 10^{15}} \cong 1.6 \cdot 10^{-4} \mathrm{MeV}=160 \mathrm{eV} .
$$

One can imaging a situation, however, in which dependence of frequency of radiation on amplitude can be useful for selection of beam amplitudes determined for processing. In Optical Stochastic Cooling, OSC, for example, this might be a specific tuning for cooling the particles with large amplitudes only. As optical amplifiers are working within $\hbar \omega_{\max } \approx 0.5-10 \mathrm{eV}$, energy, period of the wiggler must be adjusted properly, together with angle of observation.

For OSC, implementation of quadrupole like devices might be interesting for reduction of radiation from cold central part of the bunch. Typically this radiation heats the rest of the bunch and its elimination helps in cooling. This device investigated, similarly to the quadrupole 
wiggler, provides the energy shift as a function of particle displacement at location of kicker. Really the energy shift for the particle interacting with plane electromagnetic wave copropagating with the particle at the length of wiggler $L$, can be described as

$$
\Delta E=\frac{e E_{0} L}{2} \frac{K}{\gamma} \cong \frac{e E_{0} L}{2} \frac{K_{0}}{\gamma} \frac{y}{\lambda_{w}},
$$

where $E_{0}$ stands for electric field strength in co-directionally propagating electromagnetic wave. One can see that action is also linearly dependent of position of particle in the gradient undulator or in quadrupole wiggler.

\section{CONCLUSIONS}

The device analyzed -gradient undulator/wiggler can be interesting not only for the purposes of beam size measurements, but also for nonlinear correction of magnetic properties in the damping ring or in a transport channel, acting in one particular direction only. Minimal number of poles for this purposes -three-give closed bump.

Device can be interesting for implementation into OSC, serving as a pickup and/or a kicker linearly (re)acting to the particle's instant position in one direction.

\section{REFERENCES}

[1] A.A. Mikhailichenko, M.S. Zolotorev, Optical Stochastic Cooling, SLAC-PUB-6272, Jul 1993. 11pp., Phys.Rev.Lett.71: 4146-4149,1993.

[2] E.G. Bessonov, A.A. Mikhailichenko, Alignment of the Linac With the Help of Radiation from the Quadrupoles of the Linear Collider, $4^{\text {th }}$ European Particle Accelerator Conference (EPAC 94), London, England, 27 Jun - 1 Jul 1994: Proceedings Edited by V. Suller and Ch. Petit-Jean-Genaz. River Edge, N.J., World Scientific, 1994, vol. 3, p. 2579-2581.

[3] E.G. Bessonov, J. Pflüger, G.-A. Voss, N.J. Walker, Internal DESY report M96-18, September 1996.

[4] E.G. Bessonov, N.J. Walker, S.G. Wipf, Beam size measurements in Linear Collider using Gradient Undulator and off-axis Detection, TESLA 97-18, November 1997.

[5] K. Steffen, High Energy Beam Optics, Interscience Publishes, 1965.

[6] A.A. Mikhailichenko, 3D Electromagnetic Field: Representation and Measurements, Cornell CBN-95-16, 1995. 42pp.

[7] G.Dudnikova, V.Vshivkov, K.Vshivkov, $U M K A-V G$, Institute of Computation Technologies, Lab. of Plasma Physics, Siberian Branch of RAN.

[8] See for example J.D Jackson, Classical Electrodynamics, Third Edition, 1998. 